\title{
Primary hyperaldosteronism: a rare cause of acute aortic dissection
}

Heung Yong Jin and Kyung Ae Lee

Division of Endocrinology and Metabolism, Department of Internal Medicine, Research Institute of Clinical Medicine of Jeonbuk National University-Biomedical Research Institute of Jeonbuk National University Hospital, Jeonju, Korea
Received: April 6, 2020

Revised : June 4, 2020

Accepted: June 8, 2020

\section{Correspondence to} Kyung Ae Lee, M.D.

Tel: $+82-63-250-2749$

Fax: +82-63-254-1609

E-mail: kaleey@jbnu.ac.kr https://orcid.org/0000-00033700-8279
A 42-year-old woman presented to the emergency department with acute-onset severe abdominal pain. Hypertension had been diagnosed 3 years previously, which was well controlled with amlodipine $5 \mathrm{mg}$ and valsartan 80 mg. On arrival, her blood pressure was 140/80 mmHg. Serum potassium was $2.3 \mathrm{mmol} / \mathrm{L}$ and arterial blood $\mathrm{pH}$ and $\mathrm{HCO}_{3}$ were 7.468 and $27.5 \mathrm{mmol} / \mathrm{L}$, respectively. Electrocardiogram revealed normal sinus rhythm and left ventricular systolic function was normal on transthoracic echocardiography. Contrast-enhanced computed tomography revealed aortic dissection (Stanford type B) with dilated infra-renal abdom- inal aorta (Fig. 1). Additionally, a mass approximately $10 \times 17 \mathrm{~mm}$ in size was incidentally detected in the right adrenal gland (Fig. 2A). Adrenal function tests showed: plasma renin activity 0.16 $\mathrm{ng} / \mathrm{mL} / \mathrm{hr}$, aldosterone $17.7 \mathrm{ng} / \mathrm{dL}$, aldosterone: renin ratio 110 (<30). Saline infusion test was performed and aldosterone level after infusion was 29.7 (< 10) ng/dL. The patient was diagnosed with primary hyperaldosteronism (PA) caused by adrenal adenoma. After adenoma removal, serum potassium normalized and blood pressure was well controlled despite reduction in antihypertensives. Adrenal adenoma was confirmed by histopathology (Fig. 2B
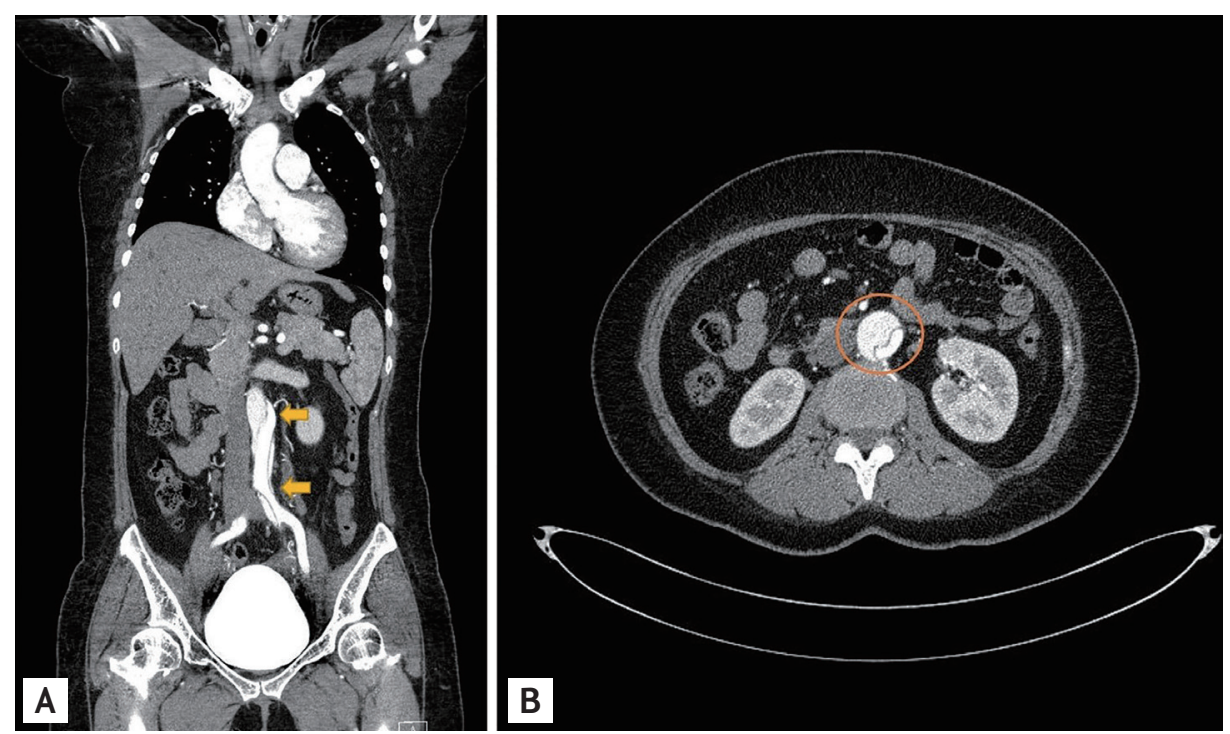

Figure 1. Spiral computed tomography (CT) of aorta. Coronal (A) and axial (B) CT scans showing a Stanford type B aortic dissection of descending aorta (yellow arrows) with dilated infra-renal abdominal aorta (yellow circle). 

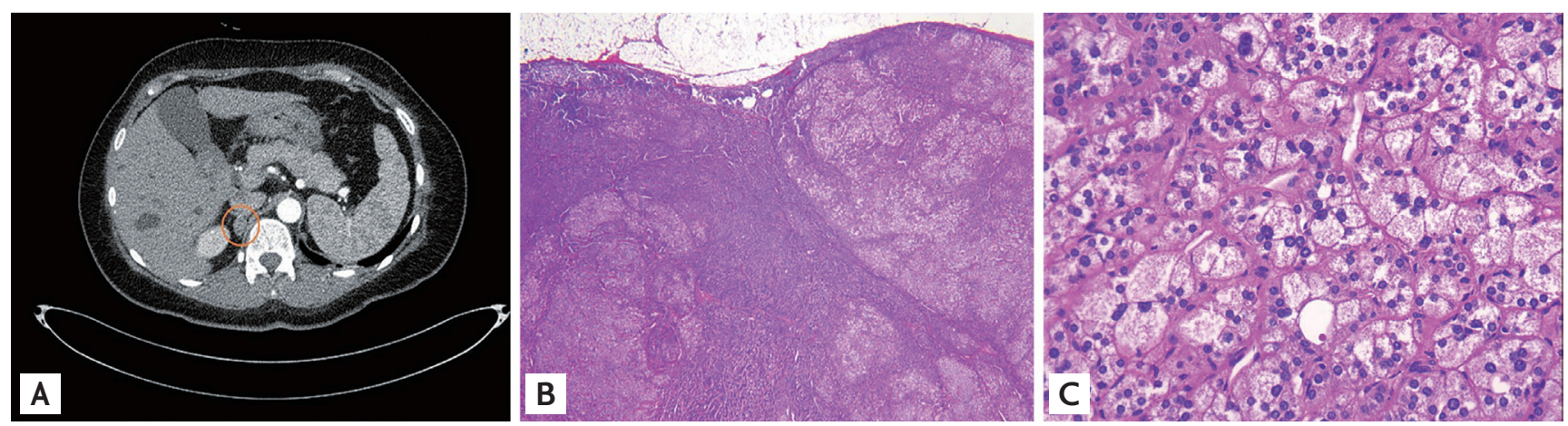

Figure 2. Abdomen computed tomography (CT) and microscopic findings of adrenal adenoma. (A) A mass (yellow circle) approximately $10 \times 17 \mathrm{~mm}$ in size was detected incidentally in the right adrenal gland. (B) Adrenal mass showing margination and thin fibrous capsule in low power field $(\mathrm{H} \& \mathrm{E}, \times 2 \mathrm{O})$. (C) The tumor cells are arrange in small nests and have finely vacuolated abundant cytoplasm without significant nuclear atypia $(\mathrm{H} \& \mathrm{E}, \times 400)$.

and $2 \mathrm{C}$ ). Hypertension and atherosclerosis remain the commonest predisposing factors for dissecting aortic aneurysm. Therefore, it is most frequently seen between the $5^{\text {th }}$ and 7 th decades following longstanding degenerative hypertensive changes. Previously, PA was considered a rare cause of secondary hypertension. However, recent developments in diagnostic tests have revealed a higher incidence than previously thought. Moreover, recent studies have demonstrated possible vasculotoxic effects of hyperaldosteronism on arterial structure, with remodeling, fibrosis, proliferation, and modification of the collagen and elastin content, independent of the blood pressure. Following accurate diagnosis, surgical resection of an adenoma or medical treatment with an aldosterone antagonist for bilateral adrenal hyperplasia can lead to favorable clinical outcomes. From our experience, it is reasonable to consider PA as a possible cause of aortic dissection.

\section{Conflict of interest}

No potential conflict of interest relevant to this article was reported. 